\title{
VIDEO STREAMING OVER WIRELESS NETWORKS
}

\author{
Xiaoqing Zhu and Bernd Girod \\ Information Systems Laboratory, Stanford University \\ Stanford, CA 94305, USA \\ \{zhuxq,bgirod\}@stanford.edu
}

\begin{abstract}
Video streaming over wireless networks is compelling for many applications, ranging from home entertainment to surveillance to search-and-rescue operations. Interesting technical challenges arise when the unpredictable nature of the wireless radio channel meets the requirements of high data rate and low latency for video transport.

This tutorial provides an overview of the technical challenges of video streaming over wireless networks, with a focus on novel cross-layer design solutions for resource allocation. Performance comparison of various centralized and distributed schemes are presented, using video streaming over wireless home networks as an application example.
\end{abstract}

\section{INTRODUCTION}

Video streaming over wireless networks is compelling for many applications, and an increasing number of systems are being deployed. Video streaming of news and entertainment clips to mobile phones is now widely available. For surveillance applications, cameras can be flexibly and cheaply installed, if a wireless network provides connectivity. A wireless local area network (WLAN) might connect various audiovisual entertainment devices in a home. Last, but not least, in search-and-rescue operations, real-time audiovisual communication over wireless ad-hoc networks can save lives.

While video streaming requires a steady flow of information and delivery of packets by a deadline, wireless radio networks have difficulties to provide such a service reliably. The problem is challenging due to contention from other network nodes, as well as intermittent interference from external radio sources such as microwave ovens or cordless phones. For mobile nodes, multi-path fading and shadowing might further increase the variability in link capacities and transmission error rate. For such systems to deliver the best end-to-end performance, video coding, reliable transport and wireless resource allocation must be considered jointly, thus moving from the traditional layered system architecture to a cross-layer design.

This tutorial provides an overview of the design challenges for video streaming over wireless networks, and surveys recent research efforts in the field. The paper is organized by wireless streaming problems of increasing complexity, ranging from the simple scenario of delivering a single video stream over a single wireless link (Section 2), to sharing a wireless multi-access channel among multiple video streams (Section 3) to the general case of multiple streams sharing a mesh network (Section 4). While most of the issues discussed are general, we use high-definition (HD) video streaming over 802.11a home networks as a concrete example when presenting simulation results.

\section{STREAMING OVER A SINGLE WIRELESS LINK}

As the wireless link quality varies, video transmission rate needs to be adapted accordingly. In [1], measurements of packet transmission delays at the MAC layer are used to select the optimal bit rate for video, subsequently enforced by a transcoder. The benefit of cross-layer signalling has also been demonstrated in [2], where adaptive rate control at the MAC layer is applied in conjunction with adaptive rate control during live video encoding.

Video rate adaptation can also been achieved by switching between multiple bitstreams encoded at different rates [3. 4], or truncating the bitstream from a scalably encoded representation [5]. Packets can also be dropped intelligently, based on their relative importance and urgency, utilizing the rate-distortion optimized framework introduced in [6].

The benefit of cross-layer video rate adaptation is illustrated in Fig. 1] We simulate the transmission of a single video stream over an otherwise idle 802.11a wireless link. With a nominal link speed of $54 \mathrm{Mbps}$ and a much slower transmission rate of $6 \mathrm{Mbps}$ for MAC-layer headers and control packets, the effective maximal throughput is about $40 \mathrm{Mbps}$ for video packets of 1500 bytes. The HD video sequence Harbor (1280x720p, $60 \mathrm{fps}$ ) is encoded using the H.264/AVC reference codec, with GOP length of 30 at various quality levels. Video streaming at one fixed quality level using TCP is compared against streaming on top of UDP with a video-aware application-layer transport protocol. The application-layer rate controller switches between different versions of video bitstreams according to estimated link capacity. While acknowledgment packets are sent for every received packet in TCP, the ACK frequency is reduced to once every ten received packets in the application-layer transport protocol. As a consequence, a higher video rate and quality can be supported, due to the reduction of acknowledgment

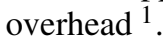

Between time 8 and 12 seconds, the simulated wireless link experiences $32 \%$ packet loss at the MAC layer, leading to many retransmissions and much lower link capacity. During this period, the transport rate of the TCP agent fluctuates over a wide range due to variations in the observed end-to-end packet round-trip-time. TCP congestion control defers transmission of incoming video packets until previous packets are acknowledged. This causes many packets to miss their playout deadline, even after the channel has recovered. When adaptation is allowed, the video bitstream is

\footnotetext{
${ }^{1}$ Since acknowledgment packets are of comparable sizes as the MAClayer control overheads, the amount of time occupied by the transmission of acknowledgment bitstreams becomes comparable to the original video stream. Therefore, per-packet acknowledgement streams may constitute a significant amount of overhead, even though their data rates are only a small fraction of the HD video streams.
} 


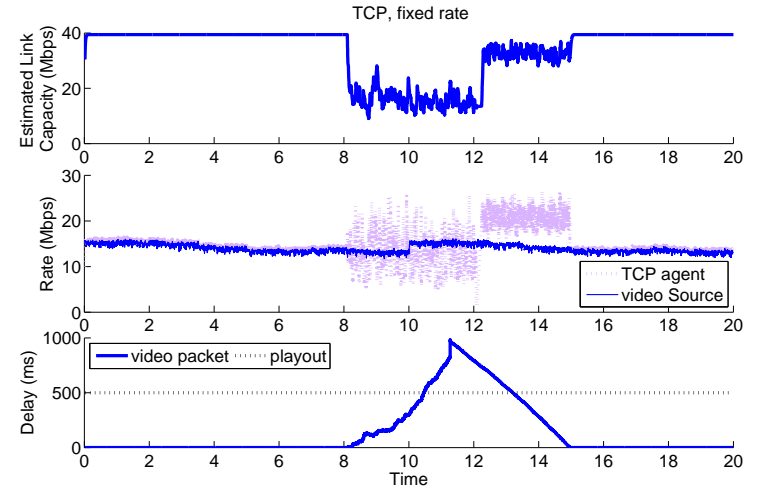

(a)

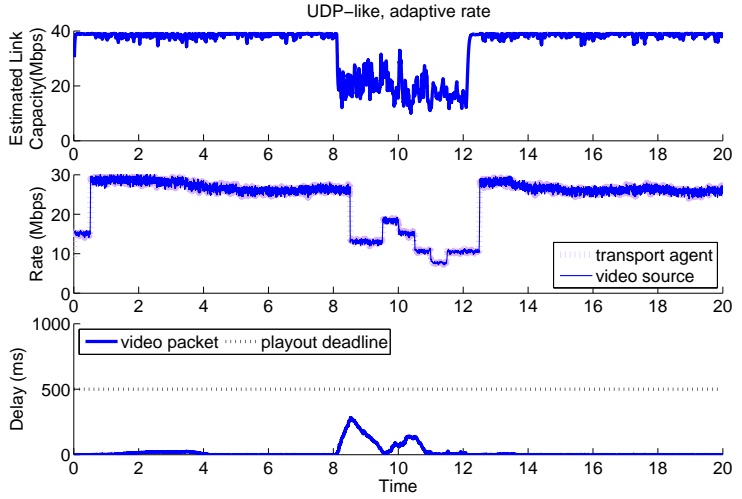

(b)

Figure 1: Comparison of video streaming over a single wireless link: a) fixed video source rate over TCP; b) adaptive video rate via bitstream switching over a video-aware application-layer transport protocol, with reduced ACK frequency. Traces are plotted for estimated link capacity in the top graphs; sending rate of the transport agents (dotted lines) and video source rate (solid lines) in the middle graphs; and packet delivery delay (measured as the time difference between the generation of a video packet and its arrival at the receiver, in solid lines) in comparison of the playout deadline (dotted line) in the bottom graphs.

switched to a version with lower rates, thereby avoiding link congestion and sustaining the video stream at a reduced quality level. In this case, the rate of the transport agent always matches that of the video source.

\section{STREAMING OVER SINGLE-HOP NETWORKS}

We now consider the scenario where multiple video streams time-share the same network over single-hop wireless connections of potentially different link speeds. Channel time allocation among the streams needs to maximize overall received video quality. The optimization can be performed jointly by a central controller when all the video streams originate from the same wireless node, e.g., the media gateway in a wireless home network or the base station of a cellular system. If, however, the video streams originate from different sending nodes, allocation needs to be carried out in a distributed fashion. This problem arises, e.g., in wireless home networks, where video might be simultaneously streamed from a DVD player, a personal video recorder and a laptop computer to different displays around the house.

\subsection{Centralized channel time allocation}

Even with centralized control, optimal channel time allocation among multiple streams is a non-trivial task. In general, the wireless links experience different channel conditions

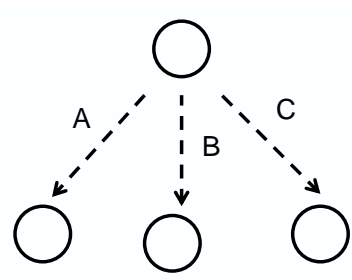

(a)

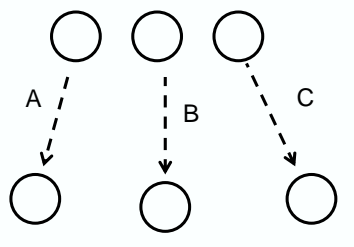

(b)
Figure 2: Network topology for multiple video streams sharing a single-hop wireless network. (a) All streams originate from the same wireless node. (b) The video source nodes are distributed. and, hence, differ in transmission speed. The video streams containing different contents also derive different utility from a change in allocated rate. As a consequence, the same allocated rate over a fast link would require lower fraction of channel time than over a slow link; the same increase in allocated rate may benefit a "hard" stream containing complex motion more than another "easy" stream with little or no motion.

In [7], channel time allocation is formulated as a convex optimization problem, with three alternative objectives: minimizing average mean-square-error (MSE) distortion of all streams (min-MSE), maximizing average PSNR of all streams (max-PSNR), and minimizing maximum MSE (minmax-MSE) among all streams. Subjective tests confirm that the min-MSE criterion corresponds best with user preferences.

Figure 2 (a) shows the network topology for comparing centralized time allocation results from the min-MSE algorithm against a heuristic scheme that divides channel time equally among all active streams. The Crew HD video sequence is streamed to three different clients over three 802.11a links at $54 \mathrm{Mbps}$ nominal link speed. Two of the wireless links are error-free, while the third link experiences $32 \%$ packet loss at the MAC layer. The estimated link capacities correspond to the maximum achievable data rate over each link, if it were allocated $100 \%$ of channel time. Traces of the estimated link capacities, resulting video rates and corresponding video qualities in PSNR are plotted in Fig. 3 Combining knowledge of the rate-distortion function of all streams, the min-MSE algorithm is able to improve the video quality traversing the worst link by $1.4 \mathrm{~dB}$ over the scheme with equal allocation, at the cost of $0.6-0.7 \mathrm{~dB}$ reduction for the other two streams.

\subsection{Distributed channel time allocation}

The multi-stream resource allocation problem becomes more challenging if it has to be solved in a distributed manner. A game-theoretic approach has been proposed for spectrum allocation among wireless stations [8]. In [9], distributed ratedistortion optimized packet scheduling is used with multiple 


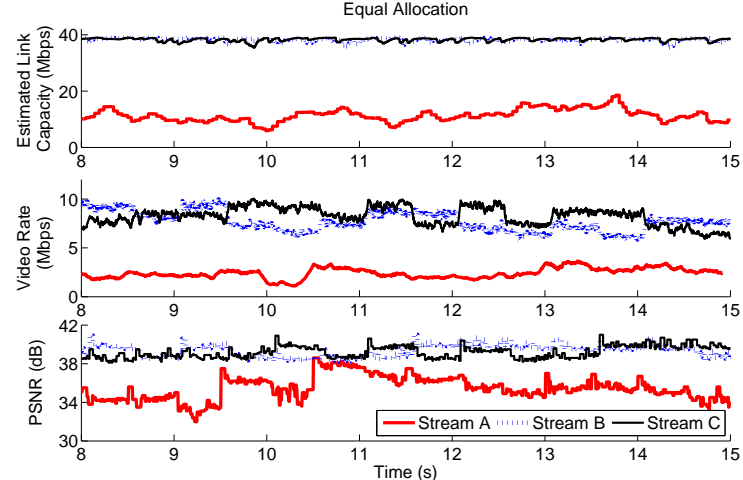

(a)
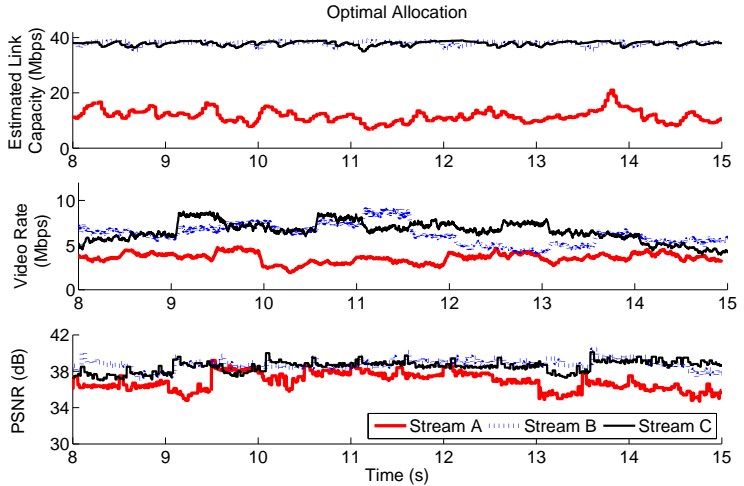

(b)

Figure 3: Centralized time allocation for three video streams sharing a WLAN (see Fig. 2 (a)): (a) equal allocation among all streams; (b) min-MSE allocation according to [7]. The total channel time constraint is set to $75 \%$ in both cases. Traces are plotted for estimated link capacity (top), resulting video rate (middle) and video quality in PSNR (bottom) for each stream. Average video PSNR for the three streams with equal channel allocation are $39.4 \mathrm{~dB}, 39.2 \mathrm{~dB}$ and $35.5 \mathrm{~dB}$ respectively. For optimized allocation, they are $38.8 \mathrm{~dB}, 38.5 \mathrm{~dB}$ and $36.9 \mathrm{~dB}$.

streams competing over a shared communication channel.

The same optimization problem as in Section 3.1 can be solved by a fully distributed protocol using a pricing mechanism. Each stream adjusts its channel time allocation according to local observations of video rate-distortion tradeoff and link capacity, as well as a common shadow price maintained at all users. The shadow price decreases when total allocation is below the given constraint to encourage rate increment from all streams, and increases when it is above the limit [10].

The efficacy of the distributed protocol is demonstrated in Fig. 4 comparing allocated rate and video quality resulting from the distributed scheme against those from an oracle-aided centralized controller. In this experiment, the Crew HD sequence is streamed from three different 802.11a nodes, all within transmission range of each other, as shown in Fig. 2(b). The nominal link speed of two of the links is fixed at $54 \mathrm{Mbps}$, while that of the third varies from $6 \mathrm{Mbps}$ to $54 \mathrm{Mbps}$. It can be observed that allocated rate achieved by the distributed scheme matches closely with the centralized solution, leading to similar video qualities. As the transmission speed of the third link approaches that of the other two, the overall video quality of all three streams improves, while the quality gap between the streams diminishes.

\section{STREAMING OVER MESH NETWORKS}

Video streaming over wireless mesh networks imposes additional challenges introduced by multi-hop transmissions. Cross-layer design and optimization for this problem is a very active area of investigation with many remaining open problems. In the following, a survey of research efforts in joint optimization of multiple protocol layers is presented first, followed by discussions on routing for media streaming, and rate allocation among multiple video streams in mesh networks.

\subsection{Multi-layer resource allocation}

The flexibility offered by cross-layer design has been exploited in a number of research efforts. Joint optimization of power allocation at the physical layer, link scheduling at the MAC layer, network layer flow assignment and transport layer congestion control has been investigated with convex optimization formulations (see, e.g., [11, 12, 13]). Our own cross-layer design framework [14] attempts to maintain a layered architecture while exchanging key parameters between adjacent protocol layers. The framework allows

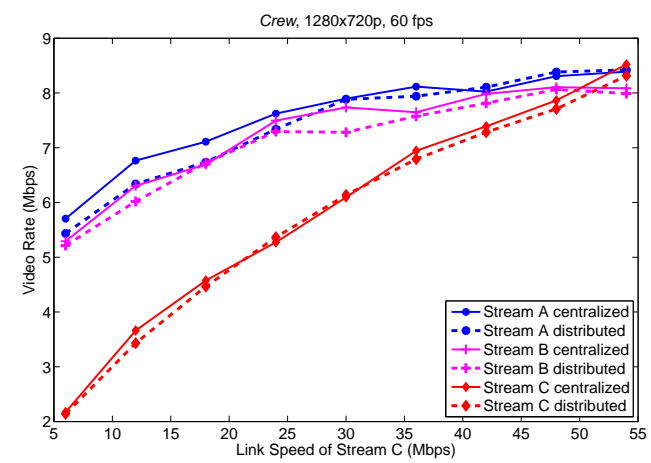

(a)

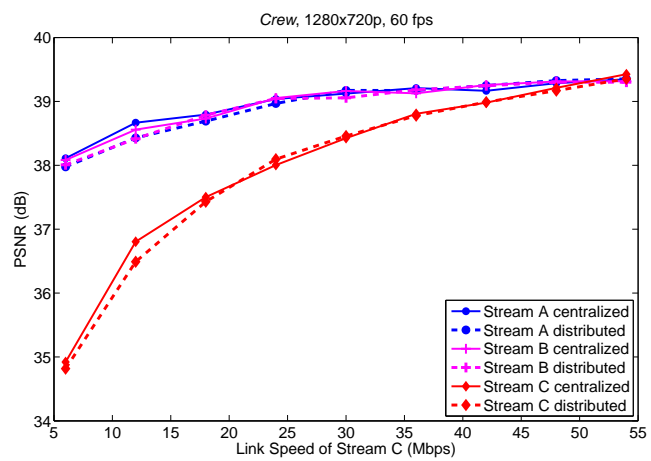

(b)

Figure 4: Channel time allocation for three video streams, all Crew, sharing a single-hop network (see Fig. 2(b)). Comparison of allocated rate (a) and resulting video quality (b) as a function of link speed of the third stream, between pricing-based distributed scheme [10] and an oracle-aided centralized controller. 
enough flexibility for significant performance gains, while keeping protocol design tractable within the layered structure, as demonstrated by the preliminary results exploring adaptive link-layer techniques, joint capacity and flow assignment, media-aware packet scheduling and congestionaware video rate allocation.

\subsection{Routing for Media Streaming}

Routing over wireless mesh networks is a difficult problem due to dynamic link qualities, even when nodes are static [15]. For video streaming, multipath routing has been proposed in combination with multiple description coding, to achieve robust delivery via path diversity [16] 17], 18].

In spite of the high data rates achieved over single-hop wireless transmissions, throughput over a multi-hop wireless path is typically significantly lower, due to contention among adjacent links along the path [19]. Since video packets need to be delivered by their playout deadline, self-inflicted congestion may drastically degrade received video quality over a throughput-limited path [20]. Route selection should therefore minimize network congestion, measured as average per-link delay of all packets. Congestion-minimized routes can be derived from solutions to a classical flow assignment problem, either via centralized computation [21] or with a distributed algorithm [22].

\subsection{Multi-Stream Rate Allocation}

When multiple streams share a wireless mesh network, their rates need to be jointly optimized to avoid network congestion while maximizing overall received video quality. The joint rate allocation problem can be solved by minimizing the Lagrangian cost of total video distortion and overall network congestion [23]. For each stream, the optimal allocated rate strikes a balance between minimizing its own video distortion and minimizing its contribution to overall network congestion. This is achieved by a distributed rate allocation protocol, which allows cross-layer information exchange between the video streaming agents at the application layer on the source nodes and the link state monitors at the MAC layer on the relay nodes.

Instead of repeating details of the distributed protocol from the original paper, we illustrate in Fig. 5 performance comparison of the proposed scheme versus TCP-Friendly Rate Control (TFRC) [24]. Two HD video sequences are streamed over a small wireless mesh network comprising five 802.11a nodes. The first stream (Harbor) travels over a 3hop path; the other ( $\mathrm{Crew}$ ) over a single-hop path. The Harbor sequence requires much higher encoding rate to achieve the same quality as $\mathrm{Crew}$, due to its more complex video contents.

Since TFRC is unaware of the video RD trade-off and relies mainly on end-to-end observations of round-trip-time and packet losses, the allocated rate for the Harbor is approximately one third of that for $\mathrm{Crew}$. This leads to around $8 \mathrm{~dB}$ of difference in the PSNR of the two received streams: while Crew is being delivered at a high quality of $39.5 \mathrm{~dB}$ in PSNR, the average quality of Harbor is only $30.9 \mathrm{~dB}$. The proposed media-aware allocation scheme, in comparison, results in increased allocation for Harbor and lower rate for Crew. Consequently, the quality gap between the two streams is reduced to $5 \mathrm{~dB}$, with Harbor improved to $31.6 \mathrm{~dB}$ and Crew remain-

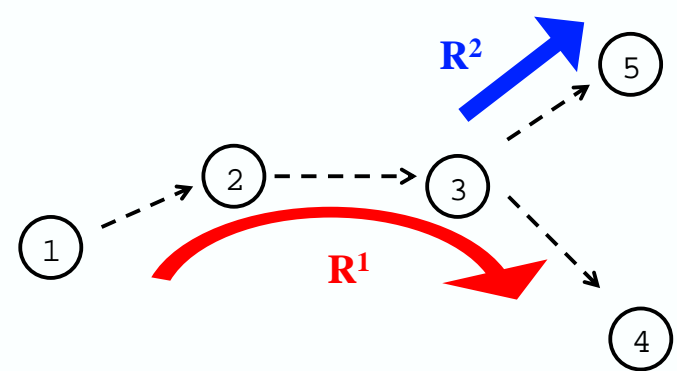

(a)

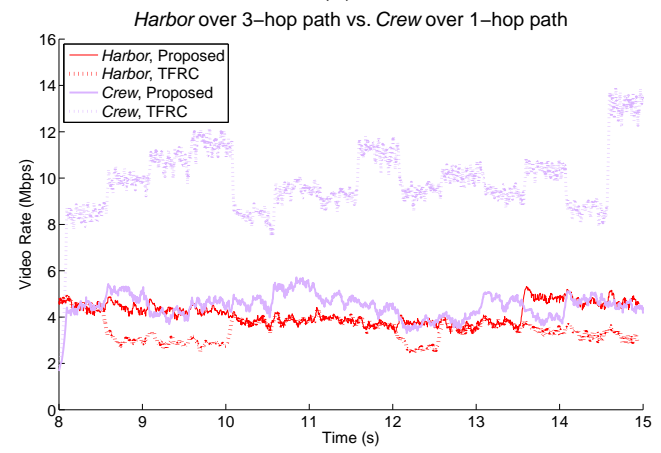

(b)

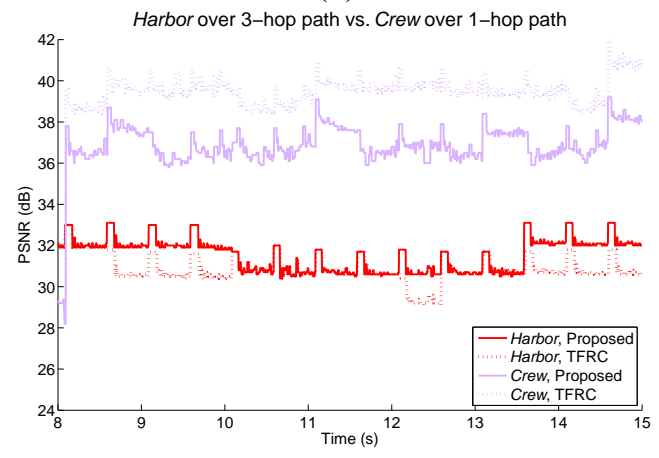

(c)

Figure 5: Two video streams competing over a wireless mesh network: (a) Network topology; (b) comparison of allocated rate resulting from the media-aware scheme in [23] versus TFRC; (c) comparison of video quality in PSNR.

ing at a relatively high quality of $37.0 \mathrm{~dB}$. It can also be noted that rate allocation from TFRC yields greater fluctuations due to traffic contention between the two streams. The cross-layer scheme, in contrast, benefits from explicit knowledge of available network throughput and maintains steady rate allocations.

\section{CONCLUSIONS AND OPEN PROBLEMS}

In this tutorial paper we have reviewed key problems and tentative solutions for video streaming over wireless networks, with an emphasis on network-adaptive rate control and resource allocation among multiple video streams. As shown in the various examples, cross-layer information exchange is required, so that video source rates can adapt to the time-varying wireless link capacities. Resource allocation 
among multiple streams can also benefit from being aware of the video characteristics (e.g., RD trade-off of each video stream) and underlying network conditions, for maximizing overall received video quality. Such considerations should be incorporated into the design of a future cross-layer protocol for video streaming over wireless networks.

Many open problems remain, particularly in the context of wireless mesh networks. For instance, it is still unclear whether the stringent latency constraint (usually less than a second) for video streaming can be met when packets need to be delivered over multiple hops of time-varying wireless links in a mesh network. Conditions where multipath routing is beneficial for streaming need to be identified, as contention of video traffic along parallel paths may cancel out the path diversity advantage of robustness to packet losses. Typically the wireless network is shared by both video streaming and other applications such as file downloading. The problem remains to be addressed as how to optimally allocate network resource among heterogeneous traffic types, each bearing a different performance metric (e.g., completion time for file downloading versus video quality for streaming).

\section{REFERENCES}

[1] P. van Beek and M. U. Demircin, "Delay-constrained rate adaptation for robust video transmission over home networks," IEEE International Conference on Image Processing, (ICIP'05), Genova, Italy, vol. 2, pp. 173-176, Sept. 2005.

[2] L. Haratcherev, J. Taal, K. Langendoen, R. Lagendijk, and H. Sips, "Optimized video streaming over 802.11 by crosslayer signaling," IEEE Communications Magazine, vol. 44, no. 1, pp. 115-121, Jan. 2006.

[3] T. Ozcelebi, M.R. Civanlar, and A.M. Tekalp, "Minimum delay content adaptive video streaming over variable bitrate channels with a novel stream switching solution," Proc. IEEE International Conference on Image Processing, (ICIP'05), vol. 1, pp. 209-212, 2005.

[4] T. Stockhammer, M. Walter, and G. Liebl, “Optimized h. 264-based bitstream switching for wireless video streaming," Proc. IEEE International Conference on Multimedia and Expo, (ICME'05), Amsterdam, The Netherlands, pp. 13961399, July 2005.

[5] F. Yang, Q. Zhang, W. Zhu, and Y.-Q. Zhang, "Bit allocation for scalable video streaming over mobile wireless internet," Proc. Twenty-third AnnualJoint Conference of the IEEE Computer and Communications Societies (INFOCOM'04), HongKong, China, vol. 3, pp. 2142 - 2151, Mar. 2003.

[6] P. A. Chou and Z. Miao, "Rate-distortion optimized streaming of packetized media," IEEE Transactions on Multimedia, vol. 8, no. 2, pp. 390-404, Apr. 2006.

[7] M. Kalman and B. Girod, "Optimal channel-time allocation for the transmission of multiple video streams over a shared channel," Proc. IEEE International Workshop on Multimedia Signal Processing (MMSP'05), Shanghai, China, pp. 1-4, Oct. 2005.

[8] A. Larcher, H. Sun, M. van der Schaar, and Z. Ding, "Decentralized transmission strategies for delay-sensitive applications over spectrum agile network," Proc. Packet Video Workshop, Dec. 2004.

[9] J. Chakareski and P. Frossard, "Rate-distortion optimized distributed packet scheduling of multiple video streams over shared communication resources," IEEE Transactions on Multimedia, vol. 8, no. 2, pp. 207-218, Apr. 2006.
[10] X. Zhu, P. van Beek, and B. Girod, "Distributed channel time allocation and rate adaptation for multi-user video streaming over wireless home networks," IEEE International Conference on Image Processing (ICIP'07), Accepted, 2007.

[11] R. L. Cruz and A. Santhanam, "Optimal routing, link scheduling and power control in multi-hop wireless networks," Proc. INFOCOM, San Francisco, California, USA, pp. 702-711, Mar. 2003.

[12] Y. Wu, P. A. Chou, Q. Zhang, K. Jain, W. Zhu, and S-Y. Kung, "Network planning in wireless ad-hoc networks : A crosslayer approach," IEEE Journal on Selected Areas in Communications, vol. 23, no. 1, pp. 136-150, Jan. 2005.

[13] Y. Xi and E. M. Yeh, "Optimal capacity allocation, routing, and congestion control in wireless network," Proc. IEEE International Symposium on Information Theory (ISIT'06), Seattle, WA, USA, pp. 2511-2515, July 2006.

[14] E. Setton, T. Yoo, X. Zhu, A. Goldsmith, and B. Girod, "Cross-layer design of ad hoc networks for real-time video streaming," IEEE Wireless Communications Magazine, vol. 12, no. 4, pp. 59-65, Aug. 2005.

[15] D. De Couto, D. Aguayo, B. Chambers, and R. Morris, "Performance of multihop wireless networks: Shortest path is not enough," Proc. ACM First Workshop on Hot Topics in Networks (HotNets-I), Princeton, New Jersey, USA, pp. 83-88, Oct. 2002.

[16] J. G. Apostolopoulos and S. J. Wee, "Unbalanced Multiple Description Video Communication Using Path Diversity," IEEE International Conference on Image Processing (ICIP'01), Thessaloniki, Greece, vol. 1, pp. 966-969, Oct. 2001.

[17] S. Mao, S. Lin, S. Panwar, Y. Wang, and E. Celebi, "Video transport over ad hoc networks: Multistream coding with multipath transport," IEEE Journal on Selected Areas in Communications, vol. 21, no. 10, pp. 1721-1737, Dec. 2003.

[18] W. Wei and A. Zakhor, "Multiple tree video multicast over wireless ad hoc networks," IEEE Trans. on Circuits, Systems and Video Technology, vol. 17, no. 1, pp. 2-15, Jan. 2007.

[19] J. Li, C. Blake, D. S. J. De Couto, H. I. Lee, and R. Morris, "Capacity of ad hoc wireless networks," in Proceedings of the 7th ACM International Conference on Mobile Computing and Networking, Rome, Italy, July 2001, pp. 61 - 69.

[20] X. Zhu, E. Setton, and B. Girod, "Congestion-distortion optimized video transmission over ad hoc networks," EURASIP Journal of Signal Processing: Image Communications, vol. 20, no. 8, pp. 773-783, Sept. 2005.

[21] E. Setton, X. Zhu, and B. Girod, "Congestion-optimized multipath streaming of video over ad hoc wireless networks," Proc. IEEE International Conference on Multimedia and Expo (ICME'04), Taipei, Taiwan, vol. 3, pp. 1619-1622, July 2004.

[22] X. Zhu and B. Girod, "A distributed algorithm for congestionminimized multi-path routing over ad hoc networks," Proc. IEEE International Conference on Multimedia and Expo (ICME'05), Amsterdam, The Netherlands, pp. 1484-1487, July 2005.

[23] X. Zhu and B. Girod, "Distribued rate allocation for video streaming over wireless networks with heterogeneous link speeds," International Symposium on Multimedia over Wireless (ISMW'07), Invited Paper, Aug. 2007.

[24] S. Floyd and K. Fall, "Promoting the use of end-to-end congestion control in the internet," IEEE/ACM Trans. on Networking, vol. 7, pp. 458-472, Aug. 1999. 